\title{
Local Structure of Fe Impurity Atoms in ZnO: Bulk
}

\section{versus Surface}

\author{
J. A. McLeod ${ }^{1 \dagger},{ }^{*}$ D. W. Boukhvalov², D. A. Zatsepin ${ }^{3,4}$, R. J. Green ${ }^{1 \ddagger}$, B. \\ Leedahl $^{1}$, L. Cui ${ }^{5}$, E. Z. Kurmaev ${ }^{3}$, I. S. Zhidkov ${ }^{3,4}$, L. D. Finkelstein ${ }^{3}$, N. V. \\ Gavrilov $^{6}$, S. O. Cholakh ${ }^{4}$, and A. Moewes ${ }^{1}$ \\ ${ }^{1}$ Department of Physics and Engineering Physics, University of Saskatchewan, 116 Science \\ Place, Saskatoon, Saskatchewan S7N 5E2, Canada \\ ${ }^{2}$ School of Computational Sciences, Korea Institute for Advanced Study (KIAS) Hoegiro 87, \\ Dongdaemun-Gu, Seoul, 130-722, Korean Republic \\ ${ }^{3}$ Institute of Metal Physics, Russian Academy of Sciences-Ural Division, 620990 Yekaterinburg, \\ Russia \\ ${ }^{4}$ Ural Federal University, 620002 Yekaterinburg, Russia \\ ${ }^{5}$ Shenzhen Graduate School, Harbin Institute of Technology, Shenzhen 518055, PR China \\ ${ }^{6}$ Institute of Electrophysics, Russian Academy of Sciences-Ural Division, 620016 Yekaterinburg, \\ Russia \\ $\dagger$ (Current Address:) College of Nano Science and Technology, Soochow University, 199 Ren-Ai \\ Rd., Suzhou Industrial Park, Suzhou, Jiangsu, 215123, China [jmcleod@suda.edu.cn] \\ $\$$ (Current Address:) Department of Physics and Astronomy, University of British Columbia, 6224 \\ Agricultural Road, Vancouver, British Columbia V6T 1Z1, Canada \\ E-mail: john.mcleod@usask.ca
}

*To whom correspondence should be addressed 


\begin{abstract}
By studying $\mathrm{Fe}$-doped $\mathrm{ZnO}$ pellets and thin films with various $\mathrm{x}$-ray spectroscopic techniques, and complementing this with density functional theory calculations, we find that Fedoping in bulk $\mathrm{ZnO}$ induces isovalent (and isostructural) cation substitution $\left(\mathrm{Fe}^{2+} \rightarrow \mathrm{Zn}^{2+}\right.$ ). In contrast to this, Fe-doping near the surface produces both isovalent and heterovalent substitution $\left(\mathrm{Fe}^{3+} \rightarrow \mathrm{Zn}^{2+}\right)$. The calculations performed herein suggest that the most likely defect structure is the single or double substitution of $\mathrm{Zn}$ with Fe, although, if additional oxygen is available, then Fe substitution with interstitial oxygen is even more energetically favourable. Furthermore, it is found that ferromagnetic states are energetically unfavourable, and ferromagnetic ordering is likely to be realized only through the formation of a secondary phase (i.e. $\mathrm{ZnFe}_{2} \mathrm{O}_{4}$ ), or codoping with $\mathrm{Cu}$.
\end{abstract}

\title{
Introduction
}

Over the last decade there has been a great deal of research in doping of the binary oxides $\mathrm{ZnO}$ and $\mathrm{TiO}_{2}$ with a small percentage (in terms of total number of atoms) of $3 d$ transition metal ions. This research was driven by several reasons: among them the search for ferromagnetism above $300 \mathrm{~K}$ in a dilute magnetic semiconductor, $\frac{112}{2}$ and the possibility of band gap engineering a semiconductor to act as a photocatalyst for water splitting,,-5$]$ and the search for a new transparent conducting oxide for photovoltaics. $\frac{617}{}$ In both cases, however, the experimental results were strongly dependent on the preparation conditions, and great discrepancies were observed between thin film and bulk materials even in cases of identical composition (see, for example, Refs. $\frac{89}{9}$ and references therein). In fact, while early theoretical treatment suggested that room-temperature ferromagnetic order should spontaneously arise in transition-metal doped $\mathrm{ZnO}$ materials, 10 subsequent experiments failed to detect any ferromagnetism over large temperature ranges. $\stackrel{11}{~ L a t e r ~ e x p e r i m e n t s ~ f o u n d ~}$ room-temperature ferromagnetic order even in undoped $\mathrm{ZnO}$, but only in thin films or nanocrystals, ${ }^{12}$ but the ferromagnetism was attributed to $\mathrm{Zn}$ vacancies, and the ferromagnetic order may or may not be stabilized by Fe-doping. 13,14 
The general problem when doping binary oxides is that both isovalent and heterovalent cation substitutions are possible, and this leads to the formation of a wide variety of structural defects including vacancies, interstitial ions, and precipitates. ${ }^{15]}$ Furthermore, predicting the properties of doped binary oxides by purely theoretical means is limited due to the lack of a model properly describing the true configurations of the structural defects. This is largely due to the fact that small scale structures are not easily detected by conventional methods — especially in thin films $\frac{16}{16}$ so computational approaches must consider a rather exhaustive range of trial geometries for defects.

In this work Fe-doped (with an Fe ion fluence of about $10^{17} \mathrm{~cm}^{-2}$ ) pellet and thin film $\mathrm{ZnO}$ are examined using a variety of x-ray spectroscopies and density functional theory (DFT) calculations. The former provides site- and symmetry-specific probing of the occupied and unoccupied states, while the latter provides specific details on the formation and couplings between different defect sites, and aid in interpreting the fine structure of the x-ray spectra. In addition to providing an empirical probe of the electronic structure over a broad energy range (i.e. the entire valence or conduction bands) local to an Fe atom, x-ray spectroscopy is also very sensitive to the valency of these atoms. ${ }^{17} \mathrm{X}$-ray spectroscopy measurements can therefore be used to test the accuracy of the electronic structures calculated using DFT with a particular choice of pseudopotentials and exchange-correlation functional, and justify using these DFT calculations to predict other material properties.

\section{Experimental and Theoretical Methods}

\section{Sample Preparation}

Pellets of polycrystalline $\mathrm{ZnO}$ were made from a $99.999 \%$ pure $\mathrm{Zn}$ target sputtered under a mixed plasma of $\mathrm{Ar}(99.999 \%)$ and $\mathrm{O}_{2}(99.999 \%)$. The distance between the target and the substrate was $8.0 \mathrm{~cm}$, the base pressure was $3 \times 10^{4} \mathrm{~Pa}$, and the flow rates of $\mathrm{Ar}(10 \mathrm{sccm})$ and $\mathrm{O}_{2}(20 \mathrm{sccm})$ were controlled by mass flow controllers. The polycrystalline pellets were around $10 \mathrm{~mm}$ in diameter and $1 \mathrm{~mm}$ to $3 \mathrm{~mm}$ thick. 
To deposit the thin films, a sapphire substrate (0001) was ultrasonically cleaned in acetone and alcohol for 10 minutes, then rinsed in deionized water, and finally dried in $\mathrm{N}_{2}$. The sapphire substrates were held at a temperature of $250{ }^{\circ} \mathrm{C}$ for 90 minutes during deposition, and the deposition was carried out at a working pressure of $2 \mathrm{~Pa}$ after pre-sputtering with $\mathrm{Ar}$ for 10 minutes. When the chamber pressure was stabilized, the radio frequency generator was set to $100 \mathrm{~W}$. The growth rate of $\mathrm{ZnO}$ thin films was $3.4 \mathrm{~nm} / \mathrm{min}$ and the typical thin film thickness was $302 \mathrm{~nm}$. The polycrystalline $\mathrm{ZnO}$ samples had a hexagonal structure with lattice parameters $a=3.250 \AA$ and $c=5.207 \AA$; XRD of these films showed there was a strong preference for the $c$ axis to be aligned vertically from the substrate surface. More details about sample preparation are available in our previous paper. ${ }^{18}$

Commercial powders of $\mathrm{ZnO}, \mathrm{FeO}$, and $\mathrm{Fe}_{2} \mathrm{O}_{3}$ (Alfa Aesar, 99.99\% purity) were also acquired for reference measurements.

\section{Ion Implantation}

Both the pellet and thin film $\mathrm{ZnO}$ samples were implanted with Fe ions under vacuum (the residual pressure was $3 \times 10^{-3} \mathrm{~Pa}$ ). A $30 \mathrm{keV}$ ion beam was generated by the source based on a cathodic vacuum arc. The arc was initiated with an auxiliary discharge in argon; this caused the pressure in the chamber during ion irradiation to increase to $1.5 \times 10^{-2} \mathrm{~Pa}$. A current density of $0.7 \mathrm{~mA} / \mathrm{cm}^{2}$ was then delivered in pulse mode with a repetition rate of $25 \mathrm{~Hz}$ and a pulse duration of $0.4 \mathrm{~ms}$. After 38 minutes of exposure, the sample had an ion fluence of $10^{17} \mathrm{~cm}^{-2}$. The samples were placed on a massive water-cooled collector during exposure. The initial temperature of the samples prior to irradiation was $20^{\circ} \mathrm{C}$, and after implantation the samples were allowed to cool under vacuum for 20 minutes. Based on our stopping and range of ions in matter (SRIM) calculations, 19 the ion implantation has a peak density of $\sim 5.5 \times 10^{22}$ atoms $/ \mathrm{cm}^{3}$ at a depth of $\sim 150 \AA$, and an average density of $\sim 1.6 \times 10^{22}$ atoms $/ \mathrm{cm}^{3}$ to a maximum depth of $\sim 650 \AA$. Finally, we note that the concentration of $\mathrm{Fe}$ atoms is perhaps too large to be considered "doping" in the sense commonly used in semiconductor physics; however we will persist in using the term "doping" in 
this manuscript and the reader is welcome to interpret this term as meaning "chemical doping" or even "chemical substitution".

\section{XPS Measurements}

X-ray photoelectron spectroscopy (XPS) core-level and valence-band measurements were made using a PHI XPS Versaprobe 5000 spectrometer (ULVAC-Physical Electronics, USA) based on a classic x-ray optic scheme with a hemispherical quartz monochromator and an energy analyzer working in the range of binding energies from 0 to $1500 \mathrm{eV}$. This apparatus uses electrostatic focusing and magnetic screening to achieve an energy resolution of $\Delta \mathrm{E} \leq 0.5 \mathrm{eV}$ for $\mathrm{Al} \mathrm{K} \alpha$ radiation $(1486.6 \mathrm{eV})$. The samples were introduced to vacuum $\left(10^{-7} \mathrm{~Pa}\right)$ for 24 hours prior to measurement, and only samples whose surfaces were free from micro-impurities were measured and reported herein. The XPS spectra were recorded using Al $K \alpha$ x-ray emission; the spot size was $100 \mu \mathrm{m}$, and the x-ray power load delivered to the sample was less than $25 \mathrm{~W}$. Typical signal to noise ratios were greater than 10000:3. Finally, the spectra were processed using ULVAC-PHI MultiPak Software 9.3 and the residual background was removed using the Tougard method. ${ }^{20}$ The XPS spectra were calibrated using a reference energy of $285.0 \mathrm{eV}$ for the carbon $1 s$ core level. ${ }^{21}$ The survey scans, shown in Figure 1, reveal a relatively low carbon content and, more importantly, show no indication of metal impurities other than Fe.

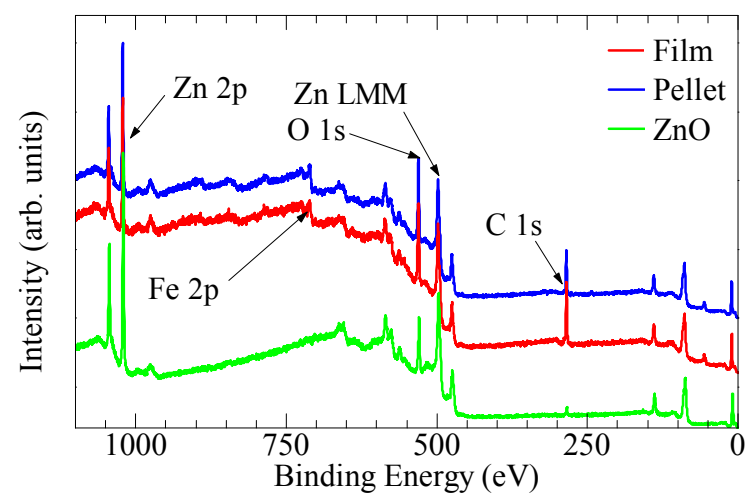

Figure 1: XPS survey scans of Fe-doped pellet and thin film $\mathrm{ZnO}$, as well as an undoped reference. The significant features are labeled. (Color online.) 


\section{XES and XAS Measurements}

X-ray emission spectroscopy (XES) and x-ray absorption spectroscopy (XAS) measurements at the Fe $L$-edge and $\mathrm{O} K$-edge were performed using Beamline 8.0.1 $1^{22}$ at the Advanced Light Source (ALS) at the Lawrence Berkeley National Laboratory. The beamline uses a Rowland circle type grating spectrometer with spherical gratings. Photons emitted from the sample were detected at an angle of $90^{\circ}$ to the incident photons, and the incident photons were $30^{\circ}$ to the sample surface normal with a linear polarization in the horizontal scattering plane. The measurements were per-

formed in a vacuum chamber at $10^{-5} \mathrm{~Pa}$, the XAS resolving power $\left(E / \Delta E\right.$ was about $2 \times 10^{3}$, while the XES resolving power was about $10^{3}$.

The Fe $L$-edge XAS spectra were calibrated using a reference energy of $708.4 \mathrm{eV}$ for the first peak in the $L_{3}$ absorption edge and a reference splitting of $13.5 \mathrm{eV}$ between the $L_{3}$ and $L_{2}$ absorption lines of metallic iron; the XES spectra were then calibrated with respect to the elastic scattering peaks from incident x-rays with energies resonant with the $L_{2}$ and $L_{3}$ absorption lines. The $\mathrm{O} K$ edge XES and XAS spectra were calibrated using a reference energy of $526.0 \mathrm{eV}$ and $532.7 \mathrm{eV}$ for the $\mathrm{O} K$ emission line and absorption edge of $\mathrm{Bi}_{4} \mathrm{Ge}_{3} \mathrm{O}_{12}$, respectively. High-resolution XAS measurements in both bulk sensitive total fluorescence yield (TFY) and surface sensitive total electron yield (TEY) modes were later performed at the Fe $L$-edge and O $K$-edge using the SGM beamline at the Canadian Light Source, $\frac{23}{2}$ these spectra were then calibrated in same way as the aforementioned XAS measurements. We point out that the absolute scale of these measurements is not strictly relevant for our discussion herein.

\section{DFT Calculations}

The density-functional theory (DFT) calculations were performed using the SIESTA pseudopotential code, $\stackrel{24 \mid 25}{2}$ in a manner similar to that previously used in related studies of impurities in semiconductors. ${ }^{26}$ All calculations were performed using the Perdew-Burke-Ernzerhof variant of the generalized gradient approximation $(\mathrm{GGA}-\mathrm{PBE})^{27}$ for the exchange-correlation potential. A full optimization of the atomic positions was performed, during which the electronic ground state 
was consistently found using norm-conserving pseudopotentials for cores and a double- $\zeta$ plus polarization basis of the localized orbitals for $\mathrm{Fe}, \mathrm{Zn}$, and $\mathrm{O}$. The forces and total energies were optimized with an accuracy of $0.04 \mathrm{eV} / \AA$ and $1 \mathrm{meV}$, respectively. For the atomic structure calculations, a $\mathrm{Zn}$ pseudopotential with $\mathrm{Zn} 3 d$ electrons treated as localized core states was employed. All calculations were performed with an energy mesh cut-off of 360 Ry and a $k$-point mesh of $6 \times 6 \times 6$ using the Monkhorst-Park scheme. 28

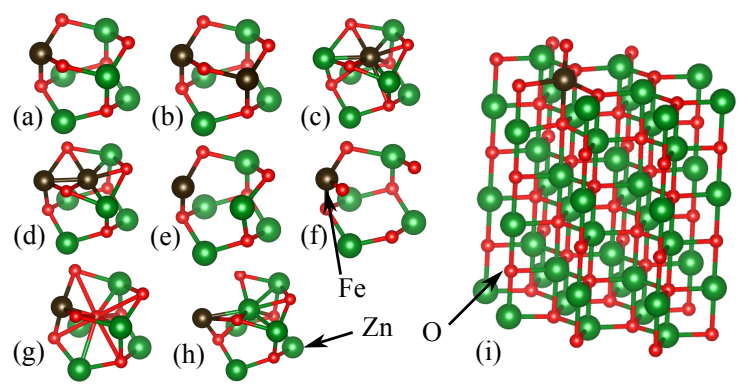

Figure 2: Optimized structures of bulk $\mathrm{ZnO}$ in the vicinity of various defects such as: (a) single iron substitution $(S)$, (b) double iron substitution $(2 S)$, (c) interstitial iron $(I)$, (d) single iron substitution and interstitial iron $(S+I)$, (e) single iron substitution and an oxygen vacancy $\left(S+V_{O}\right)$, (f) single iron substutition and a zinc vacancy $\left(S+V_{Z n}\right)$, $(\mathrm{g})$ single iron substitution with interstitial oxygen $\left(S+I_{O}\right)$, and (h) single iron substitution with interstitial zinc $\left(S+I_{Z n}\right)$. The optimized structure of a slab used for modelling surface iron substitutions passivated by oxygen is shown in (i). (Color online.)

The formation energy $\left(E_{\mathrm{form}}\right)$ calculations were performed using the standard method described in detail in Ref. ${ }^{26}$ Exchange interactions between the clusters were calculated by the formula $J=\left(E_{A F M}-E_{F M}\right) / 2 S^{2}$, where $E_{F M}$ and $E_{A F M}$ are the total energies corresponding to parallel and antiparallel orientation of spins on the remote clusters, respectively, and $S$ is the total spin of the Fe atoms. To understand the nature of the ferromagnetism in the materials, different combinations of structural defects induced by Fe ion implantation were calculated in the $\mathrm{ZnO}$ matrix. As a host for the studied defects, a $\mathrm{ZnO}$ supercell consisting of 108 atoms was used. We then performed calculations for various combinations of substitutional $(S)$ and interstitial $(I)$ Fe impurities (see Fig. 2. (a)-(h)). Point defects such as oxygen and zinc vacancies, and interstitial atoms (further denoted as $V_{O}, V_{Z n}, I_{O}$, and $I_{Z n}$ ) have also been considered herein. To model an iron impurity on the $\mathrm{ZnO}$ surface, a six layer slab was used (see Fig. 2, (i)). For more details of our computational method see 
our previous work on modelling transition metal and aluminum impurities in semiconductors. ${ }^{26}$

We would like to note that our choice of using GGA-PBE, rather than a more complex approach such as using a hybrid functional or on-site Hubbard $U$, was driven by the facts that GGA-PBE is computationally much simpler than the other approaches, GGA-PBE adequately reproduces the electronic structure of TM-doped DMS systems,, 26 and the more complex approaches provide virtually the same results in terms of defect structures and exchange interactions. ${ }^{29 \mid 30}$ More detailed theoretical analyses of defect formation (such as oxygen vacancies) in $\mathrm{ZnO}$ can be found in the literature, ${ }^{31 / 32}$ while the formation energies reported herein may not be as accurate as those determined with more sophisticated methods, the trends reported herein are essentially the same as those found elsewhere in the literature. Likewise both our DFT calculations and spectroscopy measurements suggest that the band gap of $\mathrm{ZnO}$ is reduced after Fe-doping, however neither technique is suited to accurately determining what the band gap of Fe-doped $\mathrm{ZnO}$ is (GGA is well known to underestimate band gaps,, 33 and the core hole shift in the XAS spectrum of Fe-doped $\mathrm{ZnO}$ is unknown).

\section{Multiplet Calculations}

The Fe $L$-edge RXES and XAS spectra were simulated using ligand field multiplet calculations. $\frac{3435}{35}$ For simplicity, the local symmetry of an Fe substitution was treated as tetrahedral (the true point group of $\mathrm{Zn}$ in hexagonal $\mathrm{ZnO}$ is $C_{6 v}$, but this is similar to tetrahedral). The $10 D q$ value (this value represents the splitting between the $e_{g}$ and $t_{2 g}$ bands) used in the calculations was $-0.5 \mathrm{eV}$; this was chosen by comparing the calculated spectra to the experimental measurements. The calculated spectra were broadened via a convolution with a Lorentzian profile (to incorporate the influence of life-time broadening) and a Gaussian profile (to incorporate the influence of instrumental broadening). 


\section{Results and Discussion}

\section{Spectroscopy Measurements}

X-ray spectroscopy provides a means of directly probing atomic and symmetry-selective states of a system, and is therefore a valuable tool in studying the transition metal doping of ZnO. XPS spectroscopy directly probes occupied states and can therefore be used to profile the total (occupied) density of states (DOS) in the valence band. Secondly, due to the final-state interaction between a core-hole and the valence band, core-level XPS (in the present case, the direct excitation of Fe $2 p_{1 / 2}$ or $2 p_{3 / 2}$ states) can provide information about the chemical environment of the absorbing atom. ${ }^{3436}$ In XAS spectroscopy, an x-ray is absorbed by a core level electron, this electron is consequently excited to an (unoccupied) conduction band state after the absorption of an x-ray; this transition is governed by dipole selection rules. Similarly, XES spectroscopy involves a dipole selection-driven transition from an occupied valence state to an unoccupied core level combined with the emission of an X-ray. XAS and XES can therefore probe the local partial DOS of the conduction and valence bands, respectively. Finally, when an XES spectrum is taken when the excitation energy is resonant with an absorption feature, resonant inelastic x-ray scattering (RIXS) can occur. This changes the shape of the XES spectrum when compared to the non-resonant case, and these resonant excitations offer a local probe of magnetic, multiplet, and charge-transfer transitions. 37

The XPS core and valence level spectra for Fe-doped and pure $\mathrm{ZnO}$ are shown in Fig. 3. The local structure of the iron impurities is studied by XPS Fe $2 p$-core level spectra, as shown in Fig. 3 (i). The $2 p_{1 / 2}$ and $2 p_{3 / 2}$ peaks are clearly visible, and each has an accompanying satellite line. It is

clear that the Fe-doped $\mathrm{ZnO}$ XPS spectrum in the pellet is quite close to that of $\mathrm{FeO},{ }^{\sqrt[36]{6}}$ indicating that the Fe-doping in the pellet is primarily due to isovalent substitution (i.e. $\mathrm{Fe}^{2+}$ ). On the other hand, the spectrum from the Fe-doped $\mathrm{ZnO}$ thin films resembles something in between the $\mathrm{Fe}^{2+}$ in $\mathrm{FeO}$ and the $\mathrm{Fe}^{3+}$ in $\mathrm{Fe}_{2} \mathrm{O}_{3}$. This suggests that the iron in the $\mathrm{Fe}$-doped $\mathrm{ZnO}$ thin film is a mixture of both $\mathrm{Fe}^{2+}$ and $\mathrm{Fe}^{3+}$, indicating that likely both isovalent and heterovalent substitutions occur. 
This finding is in agreement with previous studies. ${ }^{14}$ The shapes and satellite lines in the spectra from $\mathrm{FeO}$ and $\mathrm{Fe}_{2} \mathrm{O}_{3}$ are well known in the literature., $34[38,40$

By subtracting $70 \%$ of the pellet Fe $2 p$ XPS spectrum from the thin film Fe $2 p$ XPS spectrum a "difference spectrum" is produced and shown in Fig. 3 (ii); the features in this spectrum are energetically aligned with those of $\mathrm{Fe}_{2} \mathrm{O}_{3}$. This qualitative treatment suggests that the $\mathrm{Fe}$-doping in thin film $\mathrm{ZnO}$ is approximately $30 \% \mathrm{Fe}^{3+}$ and $70 \% \mathrm{Fe}^{2+}$, to within the probe depth of XPS (some justification and discussion of these values may be found at the end of this section). Charge compensation for $\mathrm{Fe}^{3+}$ substitution can be realized by $\mathrm{Zn}$ vacancies, or interstitial oxygen. We should add that from these measurements we cannot entirely rule out the presence of trace amounts of $\mathrm{Fe}^{3+}$ on the surface of the $\mathrm{ZnO}$ pellets (for example, one could claim that the $2 p$ peaks in the XPS spectrum from the pellet are slightly higher in binding energy than those in the spectrum from $\mathrm{FeO}$, as shown in Fig. 3(i)), however by comparing the shape of the Fe $2 p$ XPS spectra of $\mathrm{FeO}, \mathrm{Fe}_{2} \mathrm{O}_{3}$, and $\mathrm{Fe}$-doped $\mathrm{ZnO}$ pellets, we estimate that less than $10 \%$ of the $\mathrm{Fe}$ sites probed in Fe-doped $\mathrm{ZnO}$ pellet are $\mathrm{Fe}^{3+}$. However we stress that the presence of any $\mathrm{Fe}^{3+}$ on the surface of these pellets would increase the estimated concentration of $\mathrm{Fe}^{3+}$ in the Fe-doped thin film $\mathrm{ZnO}$ discussed above.

The XPS valence band spectra of pure and Fe-doped $\mathrm{ZnO}$ are shown in Fig. 3 (ii). The $\mathrm{Zn}$ $3 d^{10}$ shell is located at $\sim 10.9 \mathrm{eV}$, but the majority of the valence band (between 5 and $9 \mathrm{eV}$ ) is due to the bonding of $\mathrm{O} 2 p$ states. Fe-doping introduces states near the Fermi level (see Fig. 3 (iii)), that is, at energies above where the top of the valence band would be in pure $\mathrm{ZnO}$. A mid-gap feature due to Fe $3 d$ states appears at $\sim 1.5 \mathrm{eV}$ (labelled $a$ in Fig. 3(iii)) in both the ZnO pellet and thin film, while a secondary feature appears at $\sim 0.5 \mathrm{eV}$ (labelled $b$ in Fig. 3 (iii)) in the thin film sample. The former feature is likely a consequence of $\mathrm{Fe}^{2+}$ substitution, and the appearance of this feature inside the nominal band gap of $\mathrm{ZnO}$ is consistent with the general strategy of band gap engineering of $\mathrm{ZnO}$ and $\mathrm{TiO}_{2}$-based photocatalysts wherein transition metal $d^{n}$ states substitute the host $d^{10}$ or $d^{0}$ states, respectively. ${ }^{41}$ On the other hand, the latter feature is likely a consequence of $\mathrm{Fe}^{3+}$ substitution, and the appearance of these states close to the Fermi level is usually considered 
the main reason for the appearance of ferromagnetism in $\mathrm{ZnO}: \mathrm{Fe}$ thin films. $\frac{42}{42}$

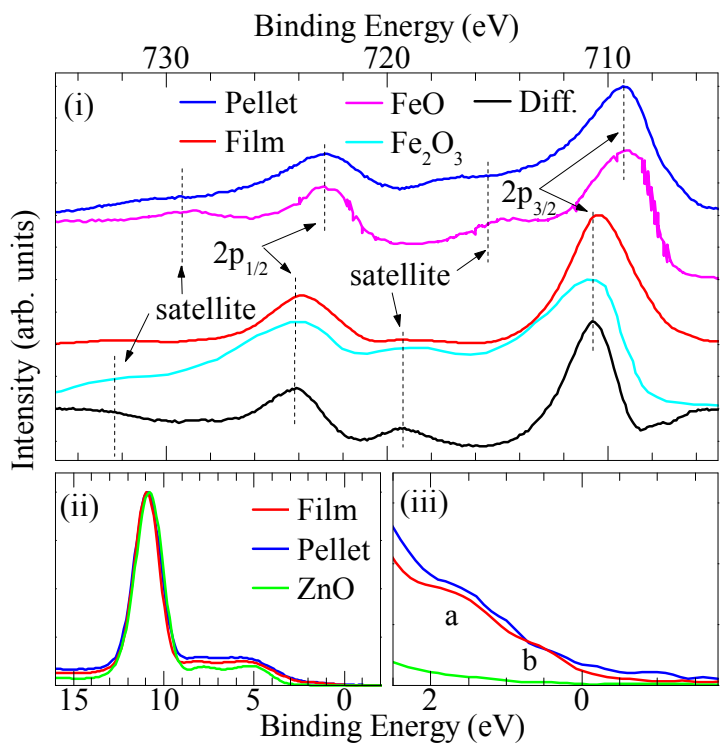

Figure 3: XPS measurements of Fe-doped ZnO: (i) The Fe $2 p$ XPS spectra from a Fe-doped $\mathrm{ZnO}$ pellet and thin film, $\mathrm{FeO},{ }^{40} \mathrm{Fe}_{2} \mathrm{O}_{3}, \stackrel{34}{ }$ and a "difference spectrum" obtained by subtracting $70 \%$ of the pellet spectrum from the thin film spectrum. (ii) The XPS VB spectra of Fe-doped pellet, thin film, and pure $\mathrm{ZnO}$. (iii) An enlarged view of the VB spectra near the band gap, showing the mid-gap valence structures in the Fe-doped samples. (Color online.)

The XAS measurements of the Fe $L$-edge in the two Fe-doped $\mathrm{ZnO}$ samples are shown in Fig. 4 (i). The bulk-sensitive TFY XAS spectra for both the pellet and thin film samples are effectively the same; this suggests that the $\mathrm{Fe}^{3+}$ observed in the thin film is primarily at the surface. The TFY XAS is also in good agreement with the calculated spectrum of tetrahedrally-coordinated $\mathrm{Fe}^{2+}$ with a $10 D q$ splitting of $-0.5 \mathrm{eV}$ (see Fig. 4 (i)). This provides additional confirmation that $\mathrm{Fe}^{2+}$ simply substitutes for $\mathrm{Zn}$ in the wurtzite lattice, and does not significantly distort the structure. The shape of these spectra is also close to that from FeO, as shown in Fig. 4(i). The surface-sensitive TEY XAS is less straightforward, this is because the surface iron can adopt different local geometries, different ligand coordinations, and is more prone to oxidation. The shape of the TEY XAS spectra from both Fe-doped pellet and thin-film $\mathrm{ZnO}$ resemble a mixture of the spectra from both $\mathrm{FeO}$ and $\mathrm{Fe}_{2} \mathrm{O}_{3}$, as shown in Fig. 4(i). Since the XAS and RIXS measurements were performed some time after the XPS measurements, and the surface of these samples was not cleaved or sputtered 
prior to measurement, it is likely that prolonged exposure to air leads to further oxidation of the surfaces of both of these samples, consequently the TEY XAS observation of a more $\mathrm{Fe}^{3+}$ rich surface compared to the XPS measurements is not surprising. Again, however, if $70 \%$ of the TEY XAS spectrum from the Fe-doped $\mathrm{ZnO}$ pellet is subtracted from the TEY XAS spectrum of the Fe-doped $\mathrm{ZnO}$ thin film $\mathrm{ZnO}$ the resulting different is almost exactly the same shape as the $\mathrm{Fe}_{2} \mathrm{O}_{3}$ spectrum, as shown in Fig. 4(i).

The Fe $L$-edge in XES measurements are shown in Fig. 4 (ii). Like the TFY XAS, the RIXS present in these spectra are in good agreement with the calculated RIXS of tetrahedrallycoordinated $\mathrm{Fe}^{2+}$ with a $10 D q$ splitting of $-0.5 \mathrm{eV}$. Note that these calculations simply model the $d d$ multiplet transitions; the non-resonant emission [labelled "NXES" in Figure 4(ii)] and the charge transfer features at the low energy end of the spectrum were not taken into account in the calculations. The agreement between both the measured RIXS and XAS with the tetrahedrallycoordinated $\mathrm{Fe}^{2+}$ calculations provides strong evidence that $\mathrm{Fe}$-doping in $\mathrm{ZnO}$ provides $\mathrm{Fe}^{2+}$ substitution in the $\mathrm{Zn}$ sites.

The $\mathrm{O} K$-edge non-resonant XES and XAS measurements of pure and Fe-doped $\mathrm{ZnO}$, as well as $\mathrm{FeO}$ and $\mathrm{Fe}_{2} \mathrm{O}_{3}$, are shown in Fig. 5. The $\mathrm{O} K$-edge $\mathrm{XES}$ of Fe-doped $\mathrm{ZnO}$ looks similar to that of pure $\mathrm{ZnO}$, this is expected because the Fe-doping is relatively minimal. Because of the very different crystal structures, and the full Fe-coordination, the $\mathrm{O} K$-edge $\mathrm{XES}$ of $\mathrm{FeO}$ and $\mathrm{Fe}_{2} \mathrm{O}_{3}$ is quite different than that of the Fe-doped $\mathrm{ZnO}$ samples. The peak of the $\mathrm{O} K$ XES spectrum of the Fe-doped $\mathrm{ZnO}$ pellet is slightly lower in energy than the peak in the spectrum of pure $\mathrm{ZnO}$, this can be attributed to the influence of Fe-doping since the peaks of the spectra of $\mathrm{FeO}$ and $\mathrm{Fe}_{2} \mathrm{O}_{3}$ also occur at lower energies in comparison with the spectrum of $\mathrm{ZnO}$ (see Fig. 5).

Likewise, the $\mathrm{O} K$-edge in XAS spectra, most notably in the bulk-sensitive TFY spectra, of the Fe-doped $\mathrm{ZnO}$ samples are quite close to that of pure $\mathrm{ZnO}$. This is expected because the implantation depth is only around $10-60 \mathrm{~nm}$, while the x-ray attenuation length at the $\mathrm{O} K$-edge is a few hundred nm, and therefore the XES and TFY XAS are mostly probing pure polycrystalline $\mathrm{ZnO}$. However there is a clear pre-edge feature in the surface-sensitive TEY spectra from both the 


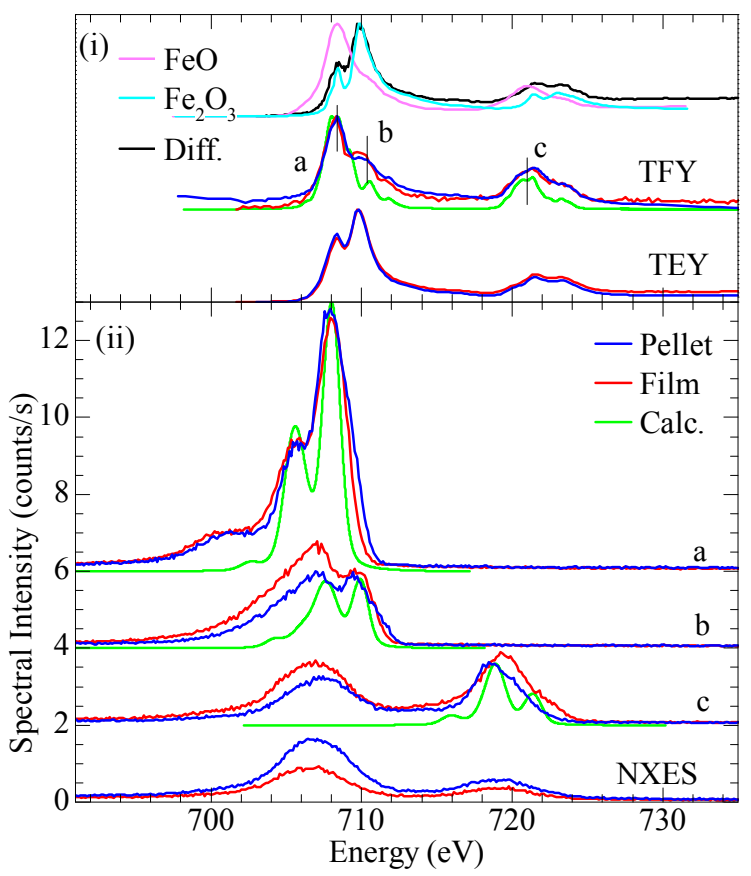

Figure 4: Fe $L$ XAS and XES measurements of Fe-doped ZnO: (i) The XAS spectra were acquired in both surface-sensitive TEY and bulk-sensitive TFY modes, here they are compared with ligandfield multiplet calculations for $\mathrm{Fe}^{2+}$, and reference spectra from $\mathrm{FeO}$ and $\mathrm{Fe}_{2} \mathrm{O}_{3}$. The letters $a, b$, $c$ denote the resonant excitation energies for the XES spectra. The normalized difference between the Fe-doped thin film TEY spectrum and 70\% of the Fe-doped pellet TEY spectrum is shown in black. (ii) The resonantly-excited XES spectra $(a, b$, and $c)$ and ligand-field multiplet calculations of $\mathrm{Fe}^{2+}$. The "NXES" spectrum was acquired with an excitation energy of $750 \mathrm{eV}$, far above resonance, and is therefore not comparable to ligand-field multiplet calculations. (Color online.) 
Fe-doped $\mathrm{ZnO}$ pellet and thin film that is similar to the pre-edge feature in the $\mathrm{O} K$-edge spectrum of $\mathrm{FeO}$. Indeed, subtracting $70 \%$ of the $\mathrm{O} K$-edge TEY spectrum from the Fe-doped $\mathrm{ZnO}$ pellet from the $\mathrm{O} K$-edge TEY spectrum of the thin film provides the "difference spectrum" in Fig. 5 , which has the characteristic two-peak pre-ege feature of $\mathrm{Fe}_{2} \mathrm{O}_{3}$. While we admit that this analysis is not precise, we have three separate measurements (Fe $2 p$-edge XPS, Fe $L_{2,3}$ TEY XAS, and O $K$-edge XAS) both suggesting that the surface of the thin film has about $30 \% \mathrm{Fe}^{3+}$ content (or at the $\mathrm{Fe}^{3+}$ content is $30 \%$ greater in the thin film than in the pellet sample).

Finally, note that the relatively low energy of the Fe-related pre-edge features in the $\mathrm{O} K$-edge $\mathrm{XAS}$ of Fe-doped $\mathrm{ZnO}$ indicates that Fe-doping reduces the band gap (at least at the surface), this was also suggested by the valence band XPS spectra. Because the $\mathrm{O} 1 s$ core hole has a very minor perturbative effect on the energy of the unoccupied states (i.e., the onset of the O $K$-edge XAS is very close to the true ground-state conduction band),, 43 the pre-edge features in the TEY spectra can be used to identify the bottom of the conduction band. Unfortunately, the Fe L-edge spectra, which provides the most direct probe of the electronic structure in the vicinity of the dopants, cannot be used to estimate the band gap because the Fe $2 p$ core hole significantly perturbs the onset of the Fe $L$-edge XAS from the true ground-state conduction band (in fact,the magnitude of this perturbation is one of the reasons why a ligand field calculation reproduces the XAS spectrum so well: the material-specific band structure is largely "washed out" and can be replaced by a single energy parameter). 44

Before discussing our DFT calculations of these structures, we would like to briefly return to the subject of the "difference spectra" we have acquired from the Fe $2 p$ XPS, Fe $L$ TEY XAS, and O $K$ TEY XAS spectra of the pellet and film samples. In all cases, we subtracted $70 \%$ of the spectrum from the pellet sample from the spectrum from the thin film sample, and concluded from the shape of the resulting "difference spectrum" compared to the corresponding spectrum of $\mathrm{Fe}_{2} \mathrm{O}_{3}$ that the thin film sample had at least $30 \%$ more $\mathrm{Fe}^{3+}$ near the surface than the pellet sample.

The value of $70 \%$ scaling for the spectrum from the pellet sample was not chosen arbitrarily. If, for pellet and thin film spectra $y_{\text {pellet }}$ and $y_{f i l m}$, we define the difference spectrum as $y_{\text {Diff }}=$ 


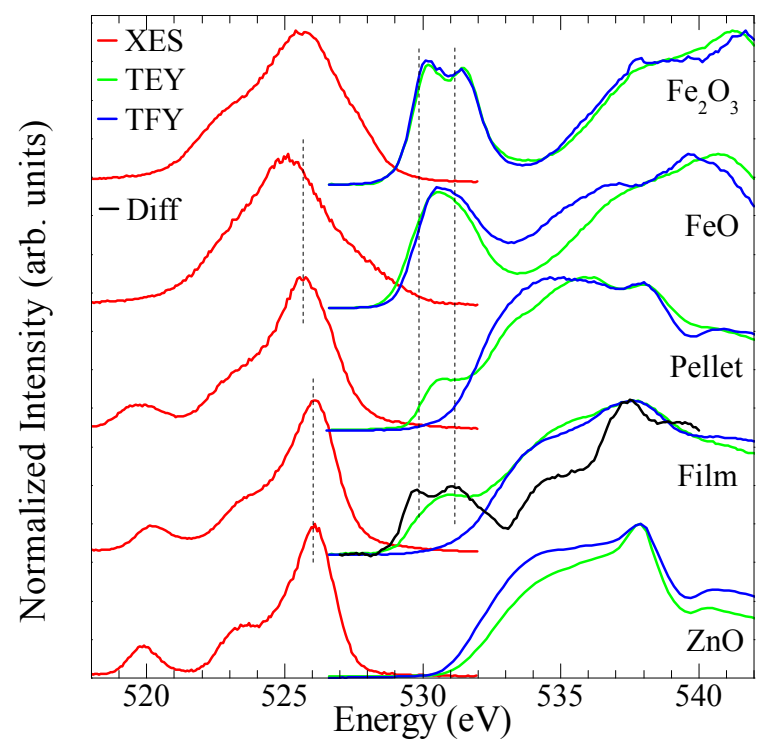

Figure 5: $\mathrm{O} K \mathrm{XES}$ and XAS measurements of Fe-doped $\mathrm{ZnO}$, pure $\mathrm{ZnO}, \mathrm{FeO}$, and $\mathrm{Fe}_{2} \mathrm{O}_{3}$. The XAS spectra were acquired in both surface-sensitive TEY and bulk-sensitive TFY modes as shown. The difference between Fe-doped $\mathrm{ZnO}$ thin film TEY spectrum and $70 \%$ of the Fe-doped $\mathrm{ZnO}$ pellet TEY spectrum is shown in black, note the resemblance to $\mathrm{Fe}_{2} \mathrm{O}_{3}$. (Color online.)

$a\left(y_{\text {film }}-\alpha y_{\text {pellet }}\right)+b$, for some renormalization constants $a$ and $b$, we can quantitatively examine the similarity between this "difference spectrum" and the appropriate spectrum from the $\mathrm{Fe}_{2} \mathrm{O}_{3}$ reference, $y_{\text {Ref }}$, as the root-mean-square (RMS) error defined in Equation ?? (where $E_{i}$ is the $i$ th energy value in the discrete spectra).

$$
\mathrm{RMS}=\sqrt{\frac{1}{N} \sum_{i}^{N}\left(y_{\mathrm{Ref}}\left(E_{i}\right)-y_{\text {Diff }}\left(E_{i}\right)\right)^{2}}
$$

The lower the RMS, the closer the "difference spectrum" is to the $\mathrm{Fe}_{2} \mathrm{O}_{3}$ reference. By choosing the renormalization constants $a$ and $b$ such that the RSS value is minimized for a particular choice of $\alpha$, we find that the best-fit value of $\alpha$ for the Fe $2 p$ XPS, Fe $L$ XAS, and O $K$ XAS are (to two significant figures) $\alpha=0.46, \alpha=0.67$, and $\alpha=0.84$, respectively. The average of these values is $\alpha_{a v g}=0.7$, which gives some justification to our claim that the thin film has $1-\alpha_{a v g}=30 \%$ or so more $\mathrm{Fe}^{3+}$ near the surface than the pellet sample.

We wish to stress that our claim of $30 \%$ more $\mathrm{Fe}^{3+}$ near the surface of the thin film sample 
than the pellet sample is still just a crude estimate, despite the justification for the value of $30 \%$ given above. Indeed, the standard deviation of the best-fit $\alpha$ values suggests that we have $\alpha_{\text {avg }}=$ $0.7 \pm 0.2$, so the claim of $30 \%$ more $\mathrm{Fe}^{3+}$ is certainly not very accurate. For simplicity, we have neglected to take the relative sharpness of the minima for each best-fit $\alpha$ into account - certainly the relative flatness of the Fe $2 p$ XPS RMS error curve in Figure 6 suggests greater intrinsic uncertainty than there is in the relatively narrow minima in the O $K$ XAS RMS error curve. However since using the RMS error between a linearly-scaled "difference spectrum" and a reference standard is hardly the definitive method for accurately quantifying the relative concentrations of $\mathrm{Fe}^{3+}$ and $\mathrm{Fe}^{2+}$, we feel justified in claiming that there is $30 \%$ more surface $\mathrm{Fe}^{3+}$ in the thin film sample compared to the pellet sample as simply a semi-quantitative estimate, and rely on this discussion to emphasize the uncertainty of this approach. In other words, we feel confident in claiming that the surface of the thin film sample is richer in $\mathrm{Fe}^{3+}$ compared to the surface of the pellet sample, but our claim that there is $30 \%$ more $\mathrm{Fe}^{3+}$ is just an attempt to quantify the previous statement, and the value of $30 \%$ contains a considerable margin of error.

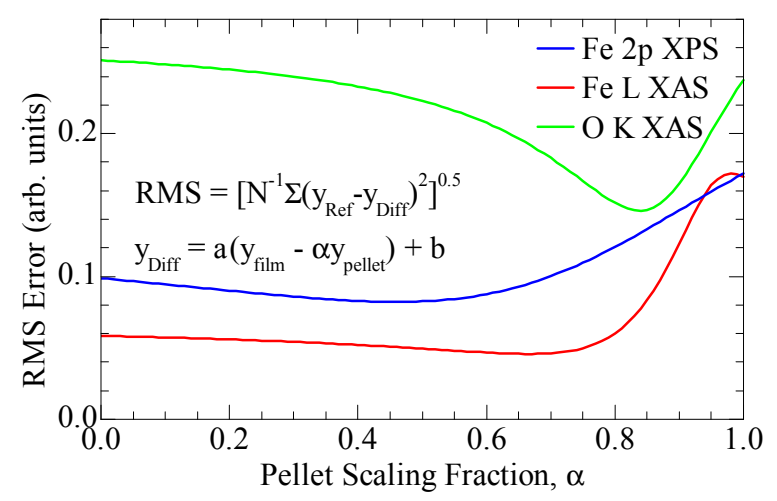

Figure 6: The root-mean-square (RMS) errors for the Fe $2 p$ XPS, Fe $L$ XAS, or O $K$ XAS "difference spectra" and the reference $\mathrm{Fe}_{2} \mathrm{O}_{3}$ spectra. (Color online.)

\section{DFT Calculations}

Our x-ray spectroscopy measurements provide a strong indication that $\mathrm{Fe}$-doping in $\mathrm{ZnO}$ shows that $\mathrm{Fe}^{2+}$ substitution occurs at $\mathrm{Zn}$ sites in the bulk of the sample; and that $\mathrm{Fe}^{3+}$ substitution 
occurs near the surface (note that both XAS in TEY mode and XPS have a probe depth of only a few nanometres). We therefore have empirical data to test the validity of our DFT structural optimizations and electronic structure calculations, and can use these calculations to gain greater insight into the influence of Fe-doping in $\mathrm{ZnO}$.

The formation energies, magnetic moments, and exchange coupling energies for the various defect structures considered herein (refer back to Fig. 2) are listed in Table 1. In agreement with our spectroscopy measurements, the lowest formation energies (with respect to pure $\mathrm{ZnO}$ ) are for isovalent single $(1 S)$ and antiferromagnetically coupled pairs $(2 S)$ of substitutional impurities, these formation energies are $+0.66 \mathrm{eV}$ and $+0.82 \mathrm{eV}$, respectively. Combined substitutional and interstitial $(S+I)$ impurities are not energetically favourable in the calculations $\left(\mathrm{E}_{\text {form }}=1.92 \mathrm{eV}\right)$; this is notable since this arrangement can be the source of long-range ferromagnetism. $\frac{45}{4}$

Table 1: Calculated formation energies $\left(\mathrm{E}_{\text {form }}\right)$ with respect to pure $\mathrm{ZnO}$, magnetic moments (M), and exchange coupling energies (J) for several defect structures. Refer back to Fig. 2 for the defect notations.

\begin{tabular}{cccc} 
Defect Type & $\mathrm{E}_{\text {form }}(\mathrm{eV})$ & $\mathrm{M}\left(\mu_{B}\right)$ & $\mathrm{J}(\mathrm{meV})$ \\
\hline $1 S$ & 0.66 & 3.90 & 0.0 \\
$2 S$ & 0.82 & 3.85 & -6.0 \\
$6 S$ & 1.40 & 3.78 & -2.0 \\
\hline $1 S+V_{O}$ & 5.59 & 3.91 & 0.0 \\
$2 S+V_{O}$ & 3.31 & 3.85 & -2.0 \\
\hline $1 S+V_{Z n}$ & 6.09 & $0.00(2+)$ & 0.0 \\
$2 S+V_{Z n}$ & 2.09 & 4.06 & +1.0 \\
$6 S+V_{Z n}$ & 1.04 & $3.92(2+), 0.64(3+)$ & $+2 \sim+4$ \\
\hline $1 S+I_{O}$ & 0.06 & 3.29 & 0.0 \\
$2 S+I_{O}$ & 0.17 & 3.91 & -1.0 \\
\hline $1 S+I_{Z n}$ & 3.60 & 3.54 & 0.0 \\
$2 S+I_{Z n}$ & 2.47 & 3.56 & -1.0 \\
$\mathrm{Fe}_{\text {surf }}$ & -2.65 & 4.07 & 0.0 \\
$\mathrm{Fe}_{\text {surf }}+\mathrm{O}$ & -4.16 & 3.15 & 0.0 \\
$I$ & 3.26 & 2.21 & 0.0 \\
$S+I$ & 1.92 & $3.76(S), 1.84(I)$ & +2 \\
$2 S+1 S(\mathrm{Cu})$ & 0.56 & 3.42 & +7
\end{tabular}

To study the nature of experimentally detected $\mathrm{Fe}^{3+}$ in thin films, calculations for various combinations of substitutional iron atoms, and various point defects have been performed. These 
calculations suggest that combinations of implanted iron with oxygen and zinc vacancies $\left(V_{O}\right.$, $V_{Z n}$ ), and interstitial zinc atoms $\left(I_{Z n}\right)$ all have large energy costs (above $2 \mathrm{eV}$ ), furthermore, these structures favour only $\mathrm{Fe}^{2+}$ based defects with magnetic moments of around $4 \mu_{B}$. As an aside, the valencies are determined by the occupancy of the Fe orbitals in our calculations - essentially the Mulliken charges 46 _ and rounding to the nearest integer. The calculated occupancy for the entire $3 d$ shell is based on an orbital radius of $2.2 \AA$, however for the radii between $1.8 \AA$ and $3.0 \AA$ the occupancy varies by only \pm 0.3 e.

Only a single substitutional iron impurity combined with interstitial oxygen $\left(1 S+I_{O}\right)$ leads to $\mathrm{Fe}^{3+}$, and this structure has a very low formation energy $(0.06 \mathrm{eV})$. One of the reasons why this structure is energetically favourable is the change in local geometry of the Fe site from the roughly tetrahedral coordination of $\mathrm{Zn}$ to something closer to an octahedral coordination that is typically found in iron oxides (see Fig. 2(g)). This configuration requires additional oxygen, while typically $\mathrm{ZnO}$ samples have oxygen deficiencies, which are caused by the low formation energy of oxygen vacancies as compared to excess interstitial oxygen. ${ }^{15}$ The calculations indicate that in Fe-doped $\mathrm{ZnO}$, the ratio of formation energies for interstitial oxygen $\left(I_{O}\right)$ and oxygen vacancies $\left(V_{O}\right)$ is just the opposite $(0.06 \mathrm{eV}$ for Fe-substitution and interstitial oxygen compared to the $5.59 \mathrm{eV}$ required for Fe-substitution and an oxygen vacancy, as shown in Table 1). Therefore, the appearance of $\mathrm{Fe}^{3+}$ can occur due to the favourable formation energies of interstitial oxygen. In addition to this, the calculations for iron substitution at the surface layer also have low formation energies $(-2.65 \mathrm{eV}$ for surface iron substitution, and $-4.16 \mathrm{eV}$ for surface iron substitution with oxygen pacification, as shown in Table 11). This energetically favourable oxidation of surface iron impurities could be the origin of $\mathrm{Fe}^{3+}$ in thin film samples, and this result is in agreement with our measurements and the measurements of others. 47

At this point we should make some comments on the "bulk" and "surface" aspects of these samples: As previously mentioned, our spectroscopic data quite plainly show a difference between the thin films and the pellets, however both of these samples are thicker than the ion implantation depth. Why then, should the surface of a Fe-doped $\mathrm{ZnO}$ thin film have significantly more $\mathrm{Fe}^{3+}$ 
sites than the surface of a Fe-doped $\mathrm{ZnO}$ pellet? The reason probably lies in the different surface geometries of these samples. The $\mathrm{ZnO}$ pellet has considerable surface roughness and no preferred orientation, while the thin film has less than $3 \mathrm{~nm}$ root-mean-square surface roughness and the polycrystalline grains strongly prefer to be oriented with the crystalline $c$-axis perpendicular to the substrate surface. ${ }^{18}$ Because of this, the average surface Fe substitution site in the thin film will quite closely resemble our DFT model structure [refer back to Fig. 2(i)], while the average surface Fe substitution site in the pellet will have no preferred geometry. Indeed, because the polycrystalline grains in the pellet would have a much rougher surface than in the thin film, we may expect that significant surface reconstruction can occur local to the surface Fe substitution sites, leading to geometries quite similar to the energetically preferred $\mathrm{Fe}^{2+}$ sites found in the bulk. In fact, it was previously found that preferential orientation of nanocrystalline grains of $\mathrm{ZnO}$ in thin films increases the chemical sensitivity of $\mathrm{ZnO}$ surfaces for vapor sensing. 48
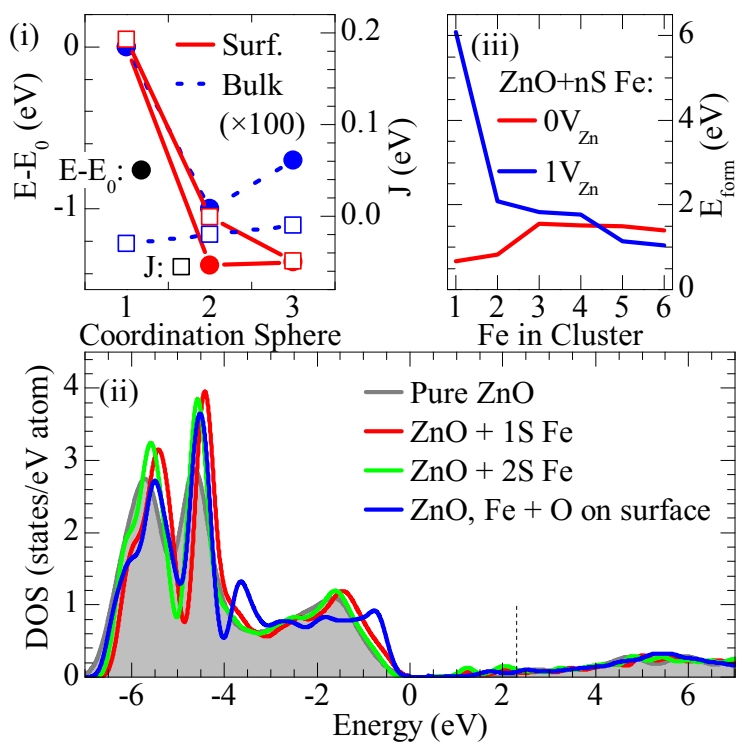

Figure 7: Cluster calculations of $\mathrm{ZnO}$ with various defects: (i) The difference in the total energies and the exchange interactions between an iron substitution on the surface and another surface iron substitution (labelled "surf"), or another iron substitution in the bulk (labelled "bulk"), as a function of the distance between the iron impurities (expressed in terms coordination spheres). (ii) The DOS for several different structures, the dotted line indicates the bottom of the conduction band for pure $\mathrm{ZnO}$ (a calculated band gap of $2.3 \mathrm{eV}$ ). (iii) The formation energy as a function of the number of substitutional iron impurities with and without a zinc vacancy. (Color online.) 
To analyze long-range super-exchange interactions for the single impurities in the presence of various point defects, the supercell was doubled along one of the axes and the exchange energies were calculated. These exchange energies were all extremely low (below $1 \mathrm{meV}$ ), and suggest the absence of long range magnetic interactions. It is therefore concluded that the iron implantation in the bulk induces $1 S$ and $2 S$ defect configurations; the former creates paramagnetic centres, while the latter creates antiferromagnetically coupled pairs, similar to codoped $\mathrm{ZnO}$ powders. $\stackrel{26}{ }$ The exchange interactions between the distance $1 S$ and $2 S$ impurities were always zero.

To investigate the possibility of substitutional iron atoms clustering in the vicinity of the $\mathrm{ZnO}$ surface, calculations on two possible types of impurity pairs were performed: the first is a twosubstitutional impurity on a surface passivated by oxygen $\left(\mathrm{Fe}^{3+}-\mathrm{Fe}^{3+}\right.$ exchange), while the second is a surface impurity passivated by oxygen and an $\mathrm{Fe}^{2+}$ substitutional impurity in the bulk. As shown in Fig. 7 (i), the first structure is quite energetically unfavourable when the second $\mathrm{Fe}^{3+}$ impurity is in the first coordination sphere of the original impurity; this suggests that $\mathrm{Fe}^{3+}$ impurities are distributed uniformly on the $\mathrm{ZnO}$ surface without any clustering. The exchange interactions for a second surface impurity in the second or third coordination sphere are antiferromagnetic $(\mathrm{J}<0$, as shown in Fig. 7(i)); ferromagnetism can only be realized by the energetically unfavourable clustering of surface iron impurities.

For the second structure, the formation energy is weakly dependent on the distance between the surface impurity and the bulk impurity, and the exchange energy is likewise weak and always antiferromagnetic (see Fig. 7. Note that the formation energies and exchange interaction energies for the "bulk" plot have been amplified by a factor of 100). The calculations therefore suggest that $\mathrm{Fe}^{3+}$ is uniformly distributed on the surface of $\mathrm{ZnO}$ (this is attractive for catalysis because the entire surface area is available), and ferromagnetism is not possible for isovalent substitution of $\mathrm{Zn}$ by Fe atoms. In fact, the latter point may help explain some recent experimental findings in which it is proposed that codoping with iron and copper is essential to the realization of ferromagnetism. $\frac{16[4950}{5}$ While it has been shown that ferromagnetic ordering is possible for some structural defects (namely $S+I$ and $2 S+V_{Z n}$ ), we have determined that these structures are not energetically 
favourable.

The calculated DOS, shown in Fig. 7(ii), suggest that Fe-doping in the $1 S$ and $2 S$ structures reduces the band gap to $0.47 \mathrm{eV}$ and $0.52 \mathrm{eV}$, respectively. This is only about $20 \%$ of the calculated gap for pure $\mathrm{ZnO}(2.3 \mathrm{eV})$. It should be pointed out that while DFT calculations are known to significantly underestimate the magnitude of the band gap, ${ }^{33}$ we expect that the qualitative trend of band gap reduction is accurate. The calculated DOS shows that hybridization between the $\mathrm{O}$ $2 p$-Fe $3 d$ states leads to a small shift in the oxygen $2 p$ band (this is also suggested in the $\mathrm{O} K$-edge XES measurements, refer back for Fig. 5), this hybridization also changes the position of the $\mathrm{Zn}$ $3 d$ band, providing the experimentally detected smearing of the corresponding peaks in the XPS valence band spectra (see Fig. 3) and the O $2 p-\mathrm{Zn} 3 d$-hybridization peak in the $\mathrm{O} K$-edge XES spectra (see the feature near $520 \mathrm{eV}$ in Fig. 5).

The appearance of ferromagnetism in Fe-doped $\mathrm{ZnO}$ has previously been attributed to a secondary magnetic phase of $\mathrm{ZnFe}_{2} \mathrm{O}_{4}$ (containing $\mathrm{Fe}^{3+}$ ). ${ }^{51}$ To examine this, the formation energies for clusters with $n$ substitutional iron impurities with and without $\mathrm{Zn}$ vacancies were calculated( $n S$, and $n S+V_{Z n}$ ). These formation energies, shown in Fig. 7(iii), indicate that for large clusters of Fe (note that this is Fe clustering in the bulk, not on the surface), $\mathrm{Zn}$ vacancies become increasingly energetically favourable. This is, for a cluster with 6 iron atoms the formation energy decreases from $1.40 \mathrm{eV}$ to $1.04 \mathrm{eV}$ per defect with a $\mathrm{Zn}$ vacancy (i.e. from $6 S$ to $6 S+V_{Z n}$ ). This makes the formation of a secondary $\mathrm{ZnFe}_{2} \mathrm{O}_{4}$ phase very probable in the iron-rich areas of doped $\mathrm{ZnO}$. The magnetic structure of a $6 S+V_{Z n}$ cluster suggests that two iron atoms convert to a $\mathrm{Fe}^{3+}$ low spin configuration, and the exchange interactions within this cluster are positive and induce ferromagnetism (see Table 1). However, for long distances (about $1 \mathrm{~nm}$ ) the exchange interaction between clusters remains negligible (less than $1 \mathrm{meV}$ ), in this way only the formation of larger sizes of similar clusters can be a source of ferromagnetism in Fe-doped $\mathrm{ZnO}$.

One other possibility for the realization of ferromagnetism in Fe-doped $\mathrm{ZnO}$ discussed in the literature is the case of copper codoping. ${ }^{49}$ To model the effect of copper codoping, single copper substitution was performed and the distance between two substituted iron defects was varied. In 

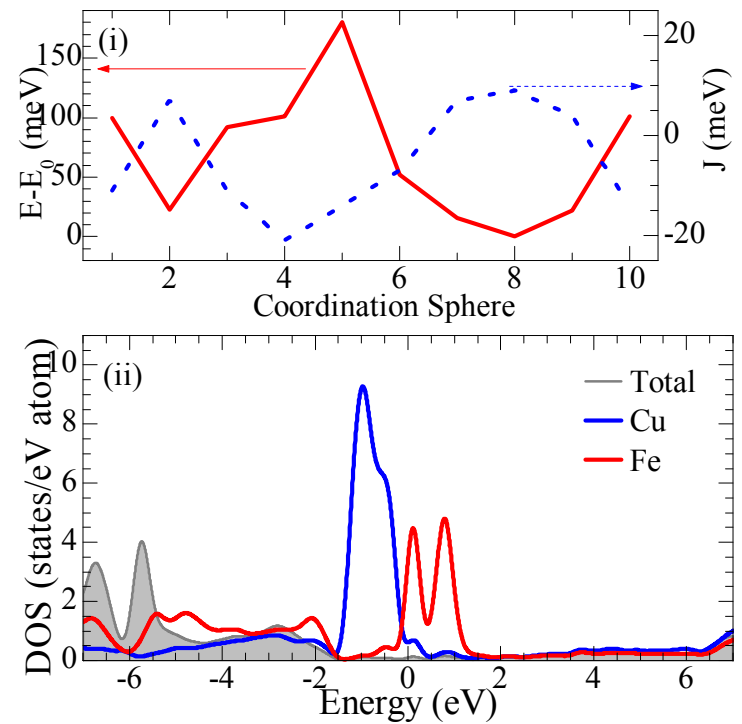

Figure 8: Cluster calculations of $\mathrm{ZnO}$ with iron and copper impurities: (i) The energy difference between the current and most energetically favourable structure and the exchange interaction as a function of the distance between iron impurities (expressed in terms of coordination spheres). (ii) The DOS for the most energetically favourable configuration of $\mathrm{ZnO}: \mathrm{Fe}, \mathrm{Cu}$. (Color online.)

this situation there was a weak variation (about $0.1 \mathrm{eV}$ ) in the formation energy of the system as a function of the change in distance between iron impurities, as shown in Fig. 8(i). However there is a substantial change in the magnetic properties, and the antiferromagnetic super-exchange interaction becomes similar to a Ruderman-Kittel-Kasuya-Yosida (RKKY) interaction (see Table 1 , and Fig. 8 (ii)). ${ }^{52}$ The RKKY interaction couples magnetic moments indirectly through conduction electrons and is the dominant exchange interaction in metals that have little overlap between magnetic electrons. The DOS of the most energetically favourable $\mathrm{Fe} \mathrm{Cu}$-codoped $\mathrm{ZnO}$ system is also significantly different from that of Fe-doped $\mathrm{ZnO}$, as shown in Fig. 8(ii). In particular, this occurs when the system is metallic and has very localized unoccupied Fe $3 d$ orbitals immediately above the Fermi level (compare to Fig. 7 (ii)). These changes occur because $\mathrm{Cu}^{1+}$ has a $3 d^{10} 4 s^{0}$ configuration (similar to $\mathrm{Zn}^{2+}$ ). This allows the formation of a single hole that should convert one of the iron atoms to a $3+$ (from the original $2+$ ) oxidation state. However, the iron defects are equivalent, so the hole is delocalized between the two, shifting the conduction band (see Fig. 8(ii) and reducing the magnetic moment of the substitutional iron impurities (see Table 1). This 
effectively creates charge carriers in the system, creating an RKKY-type exchange interaction. The calculations suggest that this is the most energetically favourable configuration of iron impurities,

and it is estimated to have a Curie temperature of $463 \mathrm{~K}$ using the Ising model $\left(T_{\mathrm{C}}=\frac{3 n}{k_{B}} J(J+1)\right.$ where $J$ is the exchange energy, $n$ is the number of exchanges, and $k_{B}$ is the usual Boltzmann constant). This Curie temperature, and the general hole-type conductivity of the system, are in good agreement with experimental results. 49

\section{Conclusions}

To summarize, we have studied the local structure of Fe-impurity atoms in pellet and thin film $\mathrm{ZnO}$ samples with various $\mathrm{x}$-ray spectroscopic techniques and DFT calculations. It is found that isovalent (and isostructural) substitution $\left(\mathrm{Fe}^{2+} \rightarrow \mathrm{Zn}^{2+}\right.$ ) takes place in both types of materials. In thin films, additional heterovalent $\mathrm{Fe}^{3+}$ substitutions (again for $\mathrm{Zn}$ ) were found primarily near the surface, and are likely due to the relatively pristine crystalline order found at the surfaces of these films. Our calculations indicate that the formation energies of single and double substitutional impurities are low, leading to the formation of paramagnetic centers or antiferromagnetic ordering, respectively. These findings assist in explaining why experimental observations of ferromagnetism are seen in Fe-doped bulk $\mathrm{ZnO}$ only after additional $\mathrm{Cu}$-doping; the reason for this is because ferromagnetic ordering occurs only in energetically unfavourable iron defect geometries.

In addition, we find that the presence of $\mathrm{Fe}^{3+}$ ions can be attributed to the formation of a secondary magnetic phase of $\mathrm{ZnFe}_{2} \mathrm{O}_{4}$, the presence of interstitial oxygen near the iron substitution site, or additional oxidation of iron defects on the surface of $\mathrm{ZnO}$. For the purpose of developing spintronic devices, Fe-doped $\mathrm{ZnO}$ is not a promising material because reproducible experimental results can only be achieved in bulk samples where ferromagnetism seems dependent on $\mathrm{Cu}$ codoping. On the other hand, Fe-doped $\mathrm{ZnO}$ may be a promising photocatalytic substrate because Fe-doping reduces the band gap; and in this regime the high level of doping used in the samples herein is appropriate. 


\section{Acknowledgement}

We gratefully acknowledge support from the Natural Sciences and Engineering Research Council of Canada (NSERC) and the Canada Research Chair program. This work was done with partial support of Ural Division of Russian Academy of Sciences (Project 12-I-2-2040) and the Russian Foundation for Basic Research (Project 13-08-00059). The Advanced Light Source is supported by the Director, Office of Science, Office of Basic Energy Sciences, of the U. S. Department of Energy under Contract No. DE-AC02-05CH11231. The Canadian Light Source is supported by NSERC, the National Research Council (NSC) Canada, the Canadian Institute of Health Research (CIHR), the Province of Saskatchewan, Western Economic Diversification Canada, and the University of Saskatchewan. DWB acknowledges the computation support from CAC of KIAS.

\section{References}

(1) Pearton, S.; Abernathy, C.; Norton, D.; Hebard, A.; Park, Y.; Boatner, L.; Budai, J. Advances in Wide Bandgap Materials for Semiconductor Spintronics. Mater. Sci. Eng., $R$ 2003, 40, $137-168$.

(2) Xu, C.; Yang, K.; Liu, Y.; Huang, L.; Lee, H.; Cho, J.; Wang, H. Buckling and Ferromagnetism of Aligned Cr-Doped ZnO Nanorods. J. Phys. Chem. C 2008, 112, 19236-19241.

(3) Ekambaram, S. Photoproduction of Clean $\mathrm{H} 2$ or $\mathrm{O} 2$ from Water using Oxide Semiconductors in Presence of Sacrificial Reagent. J. Alloys and Compd. 2008, 448, 238-245.

(4) Fujishima, A.; Honda, K. Electrochemical Photolysis of Water at a Semiconductor Electrode. Nature 1972, 238, 37-38.

(5) Catlow, C. R. A.; Guo, Z. X.; Miskufova, M.; Shevlin, S. A.; Smith, A. G. H.; Sokol, A. A.; Walsh, A.; Wilson, D. J.; Woodley, S. M. Advances in Computational Studies of Energy Materials. Philos. Trans. R. Soc. London, Ser. A 2010, 368, 3379-456. 
(6) Lee, S.-H.; Han, S.-H.; Jung, H. S.; Shin, H.; Lee, J.; Noh, J.-H.; Lee, S.; Cho, I.-S.; Lee, J.K.; Kim, J.; et al., Al-Doped ZnO Thin Film: A New Transparent Conducting Layer for ZnO Nanowire-Based Dye-Sensitized Solar Cells. J. Phys. Chem. C 2010, 114, 7185-7189.

(7) Raj, C. J.; Prabakar, K.; Karthick, S. N.; Hemalatha, K. V.; Son, M.-K.; Kim, H.-J. Banyan Root Structured Mg-Doped ZnO Photoanode Dye-Sensitized Solar Cells. J. Phys. Chem. C 2013, 117, 2600-2607.

(8) Kikoin, K. Ferromagnetic Ordering in Dilute Magnetic Dielectrics with and without Free Carriers. Journal of Magn. Magn. Mater. 2009, 321, 702-705.

(9) Hong, N. H.; Sakai, J.; Huong, N. T.; Poirot, N.; Ruyter, A. Role of Defects in Tuning Ferromagnetism in Diluted Magnetic Oxide Thin Films. Phys. Rev. B 2005, 72, 045336-5.

(10) Sato, K.; Katayama-Yoshida, H. Material Design for Transparent Ferromagnets with ZnOBased Magnetic Semiconductors. Japn. J. Appl. Phys. 2000, 39, L555-L558.

(11) Jin, Z.; Fukumura, T.; Kawasaki, M.; Ando, K.; Saito, H.; Sekiguchi, T.; Yoo, Y. Z.; Murakami, M.; Matsumoto, Y.; Hasegawa, T.; et al., High Throughput Fabrication of TransitionMetal-Doped Epitaxial ZnO Thin Films: A Series of Oxide-Diluted Magnetic Semiconductors and their Properties. Appl. Phys. Lett. 2001, 78, 3824-3826.

(12) Inamdar, D. Y.; Pathak, A. K.; Dubenko, I.; Ali, N.; Mahamuni, S. Room Temperature Ferromagnetism and Photoluminescence of Fe Doped ZnO Nanocrystals. J. Phys. Chem. C 2011, $115,23671-23676$.

(13) Hong, N. H.; Sakai, J.; Brizé, V. Observation of Ferromagnetism at Room Temperature in ZnO Thin Films. J. Phys.: Condens. Matt. 2007, 19, 036219-6.

(14) Karmakar, D.; Mandal, S. K.; Kadam, R. M.; Paulose, P. L.; Rajarajan, A. K.; Nath, T. K.; Das, A. K.; Dasgupta, I.; Das, G. P. Ferromagnetism in Fe-doped ZnO Nanocrystals: Experiment and Theory. Phys. Rev. B 2007, 75, 144404-14. 
(15) Kohan, A. F.; Ceder, G.; Morgan, D.; de Walle, C. G. V. First-Principles Study of Native Point Defects in ZnO. Phys. Rev. B 2000, 61, 15019-15027.

(16) Jeong, Y.; Han, S.; Park, J.; Lee, Y. A Critical Examination of Room Temperature Ferromagnetism in Transition Metal-Doped Oxide Semiconductors. J. Magn. Magn. Mater. 2004, 272-276, 1976-1980.

(17) de Groot, F. Multiplet Effects in X-ray Spectroscopy. Coordin. Chem. Rev. 2005, 249, 31-63.

(18) Cui, L.; Zhang, H. Y.; Wang, G. G.; Yang, F. X.; Kuang, X. P.; Sun, R.; Han, J. C. Effect of Annealing Temperature and Annealing Atmosphere on the Structure and Optical Properties of ZnO Thin Films on Sapphire (0001) Substrates by Magnetron Sputtering. Appl. Surf. Sci. 2012, 258, 2479-2485.

(19) Ziegler, J. F.; Biersack, J. P.; Ziegler, M. D. SRIM The Stopping and Range of Ions in Matter; www.srim.org, 2008.

(20) Tougaard, S. Low Energy Inelastic Electron Scattering Properties of Noble and Transition Metals. Solid State Commun. 1987, 61, 547-549.

(21) Moulder, J.; Stickle, W.; Sobol, P.; Bomben, K. In Handbook of X-ray Photoelectron Spectroscopy; Chastain, J., Ed.; Perkin-Elmer Corporation, 1992; p 261.

(22) Jia, J. J.; Callcott, T. A.; Yurkas, J.; Ellis, A. W.; Himpsel, F. J. First Experimental Results from EM / TENN / TULANE / LLNL / LBL Undulator Beamline at the Advanced Light Source. Rev. Sci. Instr. 1995, 66, 1394-1397.

(23) Regier, T.; Krochak, J.; Sham, T. K.; Hu, Y. F.; Thompson, J.; Blyth, R. I. R. Nucl. Instrum. Meth. A 2007, 582, 93-95.

(24) Ordejón, P.; Artacho, E.; Soler, J. M. Self-Consistent Order- $N$ Density-Functional Calculations for Very Large Systems. Phys. Rev. B 1996, 53, R10441-R10444. 
(25) Soler, J. M.; Artacho, E.; J, D. G.; García, A.; Junquera, J.; Ordejón, P.; Sánchez-Portal, D. The SIESTA Method for Ab Initio Order-N Materials Simulation. J. Phys.: Condens. Matter 2002, 14, 2745-2779.

(26) Chang, G. S.; Kurmaev, E. Z.; Boukhvalov, D. W.; Finkelstein, L. D.; Colis, S.; Pedersen, T. M.; Moewes, A.; Dinia, A. Effect of Co and O Defects on the Magnetism in Co-doped ZnO: Experiment and Theory. Phys. Rev. B 2007, 75, 195215-7.

(27) Perdew, J. P.; Burke, K.; Ernzerhof, M. Generalized Gradient Approximation Made Simple. Phys. Rev. Lett. 1996, 77, 3865-3868.

(28) Monkhorst, H. J.; Pack, J. D. Special Points for Brillouin-Zone Integrations. Phys. Rev. B 1976, $13,5188-5192$.

(29) García-Mota, M.; Vojvodic, A.; Abild-Pedersen, F.; Nørskov, J. K. Electronic Origin of the Surface Reactivity of Transition-Metal-Doped TiO2 (110). J. Phys. Chem. C 2012, 117, 460465.

(30) Sato, K.; Bergqvist, L.; Kudrnovský, J.; Dederichs, P. H.; Eriksson, O.; Turek, I.; Sanyal, B.; Bouzerar, G.; Katayama-Yoshida, H.; Dinh, V. A.; et al., First-Principles Theory of Dilute Magnetic Semiconductors. Rev. Mod. Phys. 2010, 82, 1633-1690.

(31) Lany, S.; Zunger, A. Assessment of Correction Methods for the Band-gap Problem and for Finite-Size Effects in Supercell Defect Calculations: Case Studies for ZnO and GaAs. Phys. Rev. B 2008, 78, 235104-25.

(32) Lany, S.; Zunger, A. Many-Body GW Calculation of the Oxygen Vacancy in ZnO. Phys. Rev. B 2010, 81, 113201-4.

(33) Dufek, P.; Blaha, P.; Schwarz, K. Applications of Engel and Vosko's Generalized Gradient Approximation in Solids. Phys. Rev. B 1994, 50, 7279-7283. 
(34) Fujii, T.; de Groot, F. M. F.; Sawatzky, G. A.; Voogt, F. C.; Hibma, T.; Okada, K. In Situ XPS Analysis of various Iron Oxide Films Grown by NO2-Assisted Molecular-Beam Epitaxy. Phys. Rev. B 1999, 59, 3195-3202.

(35) van der Laan, G.; Thole, B. T.; Sawatzky, G. A.; Verdaguer, M. Multiplet Structure in the $L_{2,3}$ X-ray-Absorption Spectra: A Fingerprint for High- and Low-Spin $\mathrm{Ni}^{2+}$ Compounds. Phys. Rev. B 1988, 37, 6587-6589.

(36) Lee, G.; Oh, S.-J. Electronic Structures of NiO, CoO, and FeO Studied by $2 p$ Core-Level X-ray Photoelectron Spectroscopy. Phys. Rev. B 1991, 43, 14674-14682.

(37) Kotani, A.; Shin, S. Resonant Inelastic X-ray Scattering Spectra for Electrons in Solids. Rev. Mod. Phys. 2001, 73, 203-246.

(38) Bocquet, A.; Mizokawa, T.; Saitoh, T.; Namatame, H.; Fujimori, A. Electronic Structure of 3d-Transition-Metal Compounds by Analysis of the 2p Core-Level Photoemission Spectra. Physical Review B 1992, 46, 3771-3784.

(39) Brundle, C.; Chuang, T.; Wandelt, K. Core and Valence Level Photoemission Studies of Iron Oxide Surfaces and the Oxidation of Iron. Surface Science 1977, 68, 459-468.

(40) Yamashita, T.; Hayes, P. Analysis of XPS Spectra of Fe2+ and Fe3+ Ions in Oxide Materials. Appl. Surf. Sci. 2008, 254, 2441-2449.

(41) Skorikov, N. A.; Korotin, M. A.; Kurmaev, E. Z.; Cholakh, S. O. Computer Simulation of the Energy Gap in ZnO- and TiO2-Based Semiconductor Photocatalysts. J. Exp. Theor. Phys. 2013, 115, 1048-1054.

(42) Potzger, K.; Zhou, S.; Reuther, H.; Mücklich, A.; Eichhorn, F.; Schell, N.; Skorupa, W.; Helm, M.; Fassbender, J.; Herrmannsdörfer, T.; et al., Fe Implanted Ferromagnetic ZnO. Appl. Phys. Lett. 2006, 88, 052508-3. 
(43) McLeod, J. A.; Moewes, A.; Zatsepin, D. A.; Kurmaev, E. Z.; Wypych, A.; Bobowska, I.; Opasinska, A.; Cholakh, S. O. Predicting the Band Gap of Ternary Oxides containing $3 d^{10}$ and $3 d^{0}$ Metals. Phys. Rev. B 2012, 86, 195207-7.

(44) Mauchamp, V.; Jaouen, M.; Schattschneider, P. Core-hole Effect in the One-Particle Approximation Revisited from Density Functional Theory. Phys. Rev. B 2009, 79, 235106-16.

(45) Jin, J.; Chang, G.; Boukhvalov, D.; Zhang, X.; Finkelstein, L.; Xu, W.; Zhou, Y.; Kurmaev, E.; Moewes, A. Element-specific Electronic Structure of Mn Dopants and Ferromagnetism of (Zn,Mn)O Thin Films. Thin Solid Films 2010, 518, 2825-2829.

(46) Mulliken, R. S. Electronic Population Analysis on LCAO-MO Molecular Wave Functions. I. J. Chem. Phys. 1955, 23, 1833-1840.

(47) Mantovan, R.; Gunnlaugsson, H. P.; Naidoo, D.; Ólafsson, S.; Johnston, K.; Masenda, H.; Molholt, T. E.; Bharuth-Ram, K.; Fanciulli, M.; Gislason, H. P.; et al., Fe Charge State Adjustment in ZnO upon Ion Implantation. J. Phys.: Condens. Matter 2012, 24, 485801-5.

(48) Wang, X.; Ding, Y.; Li, Z.; Song, J.; Wang, Z. L. Single-Crystal Mesoporous ZnO Thin Films Composed of Nanowalls. J. Phys. Chem. C 2009, 113, 1791-1794.

(49) Han, S.-J.; Song, J. W.; Yang, C.-H.; Park, S. H.; Park, J.-H.; Jeong, Y. H.; Rhie, K. W. A Key to Room-Temperature Ferromagnetism in Fe-doped ZnO:Cu. Appl. Phys. Lett. 2002, 81, $4212-4214$.

(50) Wang, Y. Q.; Su, L.; Liu, L.; Tian, Z. M.; Chang, T. Q.; Wang, Z.; Yin, S. Y.; Yuan, S. L. Ferromagnetism in Fe-doped ZnO Bulk Samples with Additional Cu Doping. Phys. Stat. Sol. (a) 2010, 207, 2553-2557.

(51) Zhou, S.; Potzger, K.; Talut, G.; Reuther, H.; von Borany, J.; Grötzschel, R.; Skorupa, W.; Helm, M.; Fassbender, J.; Volbers, N.; et al., Fe-Implanted ZnO: Magnetic Precipitates versus Dilution. J. Appl. Phys. 2008, 103, 023902-14. 
(52) Yafet, Y. Ruderman-Kittel-Kasuya-Yosida Range Function of a One-Dimensional FreeElectron Gas. Phys. Rev. B 1987, 36, 3948-3949. 